\title{
Passive tracer patchiness and particle trajectory stability in incompressible two-dimensional flows
}

\author{
F. J. Beron-Vera, M. J. Olascoaga, and M. G. Brown \\ RSMAS, University of Miami, Miami, Florida, USA
}

Received: 30 January 2003 - Revised: 4 July 2003 - Accepted: 23 September 2003 - Published: 25 February 2004

Part of Special Issue "Dedicated to Prof. A. D. Kirwan Jr. on the occasion of his 70th birthday"

\begin{abstract}
Particle motion is considered in incompressible two-dimensional flows consisting of a steady background gyre on which an unsteady wave-like perturbation is superimposed. A dynamical systems point of view that exploits the action-angle formalism is adopted. It is argued and demonstrated numerically that for a large class of problems one expects to observe a mixed phase space, i.e. the occurrence of "regular islands" in an otherwise "chaotic sea." This leads to patchiness in the evolution of passive tracer distributions. Also, it is argued and demonstrated numerically that particle trajectory stability is largely controlled by the background flow: trajectory instability, quantified by various measures of the "degree of chaos," increases on average with increasing $|\mathrm{d} \omega / \mathrm{d} I|$, where $\omega(I)$ is the angular frequency of the trajectory in the background flow and $I$ is the action.
\end{abstract}

\section{Introduction}

This paper deals with the kinematics of fluid particles in unsteady incompressible flows on the Cartesian plane. Namely, we study properties of trajectories $(x(t), y(t))$ that satisfy equations of the form

$\dot{x}=\partial_{y} \psi, \quad \dot{y}=-\partial_{x} \psi$,

where the overdot stands for time derivative and $\psi(x, y, t)$ is the streamfunction. Furthermore, we consider the latter to be split into a steady background component and an unsteady perturbation component, i.e.

$\psi=\psi^{(0)}(x, y)+\varepsilon \psi^{(1)}(x, y, t)$,

where $\varepsilon$ is a dimensionless parameter. Equation (1) constitute a canonical Hamiltonian system with $\psi$ the Hamiltonian and $(x, y)$ the generalized coordinate-conjugate momentum pair.

Two related issues are addressed in this paper. First, we investigate a cause of "patchiness" in passive tracer distributions, i.e. distributions that are mostly vigorously stirred but

Correspondence to: F. J. Beron-Vera

(fberon@rsmas.miami.edu) include poorly stirred regions (Sect. 3). Second, we study the influence of the background flow on particle trajectory stability (Sect. 4). Prior to discussing these issues, the kinematic models that we use to illustrate our results are briefly described in Sect. 2. The conclusions of the paper are given in Sect. 5.

\section{Kinematic models}

Two background flow structures in a region $[0, L] \times[0, W]$ of the $\beta$ plane are considered here. One is chosen to represent a large-scale single-gyre wind-driven ocean circulation with streamfunction given by (Stommel, 1966)

$\mathrm{S}: \psi^{(0)}=a\left[b \mathrm{e}^{b_{+} x}+(1-b) \mathrm{e}^{b_{-} x}-1\right] \sin \frac{\pi y}{W}$,

where $a:=\tau W /(\pi \lambda D), b:=\left(1-\mathrm{e}^{b_{-} L}\right) /\left(\mathrm{e}^{b_{+} L}-\mathrm{e}^{b_{-} L}\right)$, and $b_{ \pm}:=-\frac{1}{2} \beta / \lambda \pm \frac{1}{2}\left[(\beta / \lambda)^{2}+(\pi / W)^{2}\right]^{\frac{1}{2}}$. Here, $D$ is the depth, $\tau$ the wind stress amplitude (per unit density), and $\lambda$ the bottom friction. The other background streamfunction chosen corresponds to solid body rotation,

$\mathrm{R}: \psi^{(0)}=\frac{\omega_{\mathrm{R}}}{2}\left[(x-L / 2)^{2}+(y-W / 2)^{2}\right]$.

The reason for this highly idealized choice will be discussed below. Parameter values used in our numerical work are listed in Table 1.

The perturbation streamfunction is constructed by superposing standing Rossby-like modes with a power-law spectrum, namely,

$$
\begin{gathered}
\psi^{(1)}=a \sum_{k, l} A \mathrm{e}^{-\gamma x} \sin \left(k x+\phi_{k}\right) \\
\cdot \sin \left(l y+\phi_{l}\right) \cos \left(\sigma t+\phi_{\sigma}\right),
\end{gathered}
$$

where

$$
\begin{aligned}
A(k, l) & :=\frac{\pi^{2}\left(L^{-2}+W^{-2}\right)}{k^{2}+l^{2}}, \\
\sigma(k, l) & :=-\frac{\beta k}{k^{2}+l^{2}+f_{0}^{2} /(g D)},
\end{aligned}
$$




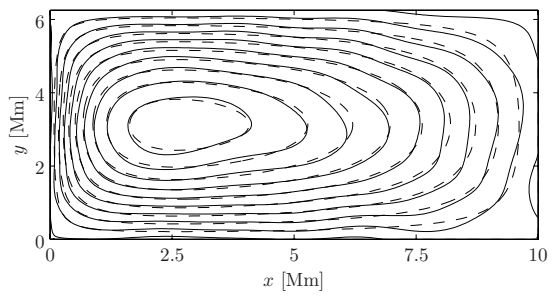

(a)

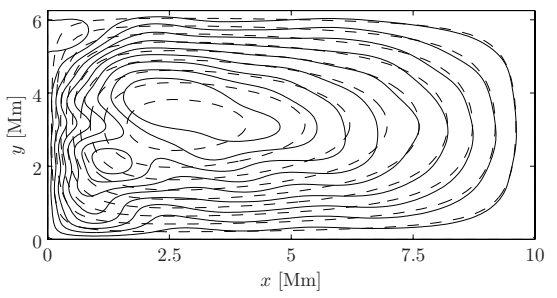

(b)

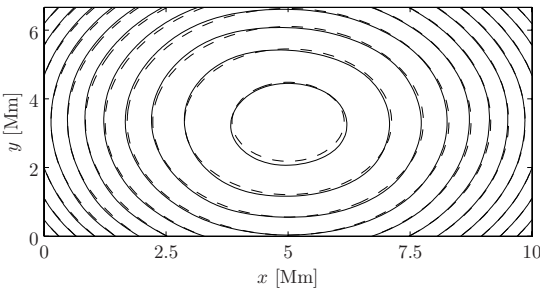

(c)

Fig. 1. Background flow streamlines (dashed lines) along with streamlines corresponding to a snapshot of the total flow at $t \approx 9 \mathrm{y}$ (solid lines). Panels (a) and (b) correspond to background flow S with different wave-like perturbation fields superimposed; panel (c) corresponds to background flow $\mathrm{R}$ with the same perturbation that was used to produce panel (a).

Table 1. Background flow parameters.

\begin{tabular}{cl}
\hline Parameter & Value \\
\hline$L$ & $10 \mathrm{Mm}$ \\
$W$ & $2 \pi \mathrm{Mm}$ \\
$D$ & $200 \mathrm{~m}$ \\
$g$ & $9.8 \mathrm{~m} \mathrm{~s}^{-2}$ \\
$f_{0}$ & $10^{-4} \mathrm{~s}^{-1}$ \\
$\beta$ & $10^{-11} \mathrm{~m}^{-1} \mathrm{~s}^{-1}$ \\
$\tau$ & $2 \times 10^{-3} \mathrm{~m}^{-2} \mathrm{~s}^{-2}$ \\
$\lambda$ & $10^{-5} \mathrm{~s}^{-1}$ \\
$\omega_{\mathrm{R}}$ & $2 \pi \mathrm{y}^{-1}$ \\
\hline
\end{tabular}

and the $\phi(k, l)$ 's are random numbers uniformly distributed between 0 and $2 \pi$. Here, $L k / \pi$ and $W l / \pi$ are positive integers; $\gamma$ is a constant; $f_{0}$ is the reference Coriolis parameter; and $g$ is the acceleration of gravity.

Dashed lines in Figs. 1a, b and Fig. 1c are streamlines for background flows $\mathrm{S}$ and R, respectively. Solid lines in these figures are total flow streamlines corresponding to a snapshot of the flow at $t \approx 9 \mathrm{y}$. The perturbation in each case involves $10 \times 10=100$ modes. In Fig. 1a and Fig. 1c the perturbation has $\varepsilon=0.05$ and $\gamma=0$. In Fig. 1b the amplitude of the $L k / \pi=1=W l / \pi$ mode is set to zero, $\phi_{k}=0=\phi_{l}$ so the flow vanishes at the boundary, $\varepsilon=0.25$, and $\gamma=0.4 \mathrm{Mm}^{-1}$.

The flows used to produce Fig. 1 and all of the numerical particle trajectory simulations presented in this paper were chosen to illustrate important aspects of Lagrangian dynamics; the flows are in many ways not representative of realistic oceanic flows. We note, however, that we focus on flows with complicated time dependence, and that the strong perturbations to the background are considered. In Fig. 1b, for example, it is seen that the perturbation leads to the presence of an eddy-like structure in the flow. Also, we note that in the flows that we have described, particle trajectories are periodic in the limit of zero perturbation strength with typical periods of about $1 \mathrm{y}$. Thus in an integration time of $10 \mathrm{y}$ most trajectories will have made approximately 10 revolutions around the gyre. The phenomena described below are not limited to ocean-basin-scale flows. In general, the trends that we describe should be evident after times in excess of a few periods of particle revolution in any background gyre flow on which a perturbation field with a broad-band of frequencies is superimposed.

\section{Passive tracer patchiness}

In this section we present numerical evidence and a theoretical argument that suggest that for a large class of systems of the form (1) phase space $(x, y)$ should be partitioned into "regular islands" in a "chaotic sea." Such a mixed phase space leads to patchiness in passive tracer distributions. $\mathrm{Nu}$ merical results are presented for a time-periodic flow $(n=1$ term in the sum in Eq. 2) and subsequently for flows with complicated time dependence ( $n$ large).

Figure 2 shows, for the time-periodic case $(n=1)$, a Poincaré section and, in the same environment, two additional trajectory diagnostics whose applicability is not restricted to time-periodic flows. The Poincare section was constructed by plotting the $(x, y)$ coordinates of several trajectories at integer multiples of the period of the streamfunction; it shows the usual mixture of "regular islands" in an otherwise "chaotic sea" (e.g. Tabor, 1989). The middle panel shows, for a dense set of trajectories with $x(0)=x_{0}$ fixed and $y(0)=y_{0}$ variable, a plot of $y$ vs. $y_{0}$ at a fixed value of $t$. The initial conditions chosen fall inside the region of the Poincaré section shown, and it is seen that both regular islands and the chaotic sea evident in the Poincaré section can be identified in the $y$ vs. $y_{0}$ plot. The same structures can also be seen in the lower panel of Fig. 2 which shows, for the same trajectories used to produce the middle panel, finite time estimates of Lyapunov exponents (described in more detail below), $v$ vs. $y_{0}$. Plots of $y$ vs. $y_{0}$ and $v$ vs. $y_{0}$ are used below to distinguish between apparently regular and apparently chaotic trajectories for flows with complicated ( $n$ large) time dependence, i.e. in flows for which a Poincaré section cannot be constructed.

Figure 3 shows plots of $y$ vs. $y_{0}$ and $v$ vs. $y_{0}$ for the nonperiodic flows used to produce Fig. 1. Trajectories in Fig. $3 \mathrm{~b}$ are 

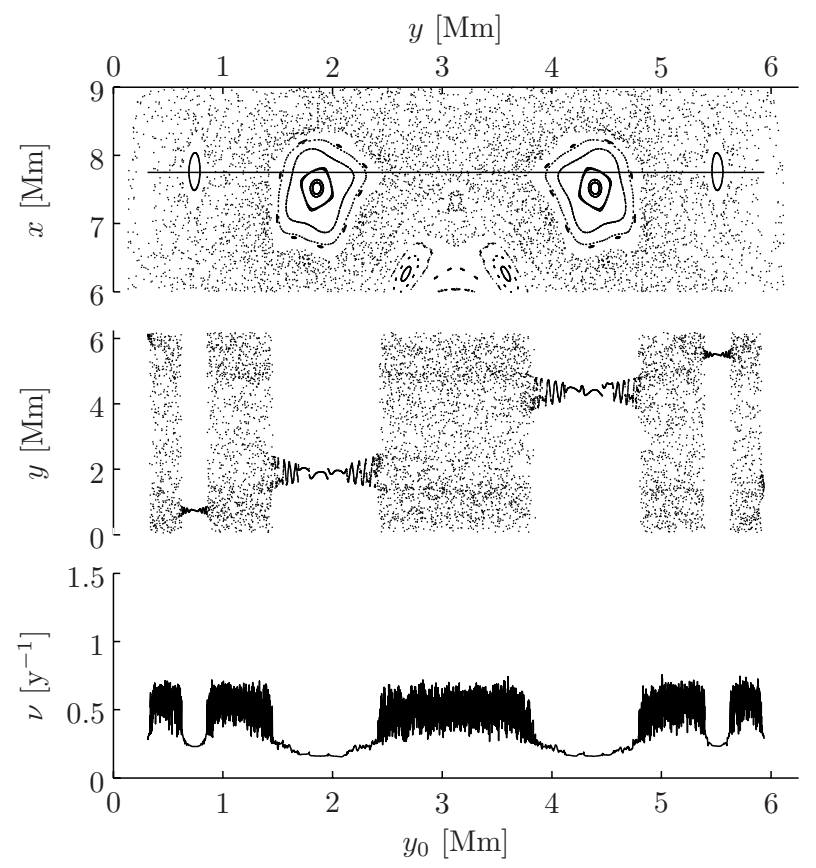

Fig. 2. Poincaré section (top), final vs. initial meridional position (middle), and finite-time estimate of the Lyapunov exponent as a function of initial meridional position (bottom). The middle and lower plots were constructed by tracking $10^{4}$ particles $\left(\Delta y_{0} \approx 5.5 \mathrm{~km}\right)$ for a duration of $150 \mathrm{y}$ in background flow $\mathrm{S}$ with a time-periodic perturbation superimposed. Particle initial positions fall on the horizontal line shown in the top panel. The perturbation parameters chosen were $\varepsilon=0.015, \gamma=0, k L / \pi=3=l W / \pi, \phi$ 's $=0$, and $2 \pi / \sigma=0.25 \mathrm{y}$.

generally more unstable than in Fig. 3a. The enhanced stability in Fig. 3a is reflected in a relatively unstructured $y\left(y_{0}\right)$ plot and smaller (on average) Lyapunov exponents than are seen in Fig. 3b. In both cases the background flow structure is the same; the difference in the stability behaviour is due to the difference in the strength of the perturbation. As expected, trajectory instability is seen to increase with increasing perturbation strength.

The difference in trajectory stability seen in Figs. 3a and c has a different explanation. The same perturbation was used in both cases, so this cannot be the cause of the difference. The cause is the influence of the background flow; this topic will be discussed in detail in the following section.

We return our attention now to Fig. 3b which corresponds to the strongly perturbed flow shown in Fig. 1b. It is seen in Fig. 3b that embedded among mostly chaotic trajectories are bands of apparently nonchaotic trajectories. These nonchaotic bands are most readily identified among the trajectories whose initial positions are near the center of the gyre; the reason for this will be discussed in the following section. Bands of nonchaotic trajectories far from the gyre center are also present, however. This is seen in Fig. 4 where two regions of Fig. $3 \mathrm{~b}$ are blown up. These apparently nonchaotic bands of trajectories are the counterparts of the "regular islands" seen in Fig. 2. Trajectories in these bands diverge only very slowly (power law dependence on time) from neighboring trajectories while chaotic trajectories diverge at an exponential rate from neighboring trajectories. The nonchaotic regions of flows are important in applications because they correspond to regions where the concentration of a passive tracer will remain high for a long duration. The existence of these regions leads to a large variance of tracer concentration or "tracer patchiness" (e.g. Pasmanter, 1988; Malhotra et al., 1998).

Another way to visualize passive tracer patchiness is offered in Figs. 5 and 6. Both figures show discrete samples of a material line of fluid at $t=0$ (vertical line segments in the figures) and at $t=12 \mathrm{y}$, in the environments shown in Fig. 1. Initial conditions in Fig. 5 are as in Fig. 3, whereas those in Fig. 6 are as in Fig. 4. These figures again show that while most of the initial material line segment is vigorously stirred, there are small portions of the initial segment, corresponding to the island-like structures seen in Figs. 3 and 4, that are poorly stirred.

We turn our attention now to explaining the occurrence of island-like structures in Figs. 2-4. First, we note that in the background flow, particle motion is describable using actionangle variables, reviewed below, and trajectories fall on tori. For perturbed systems with periodic time dependence, as in Fig. 2, it is well-known that particle trajectory dynamics are constrained by the KAM theorem (e.g. Arnold, 1989) which guarantees that for sufficiently small $\varepsilon$ some of the original tori - and associated nonchaotic motion - are preserved. Related theoretical results, generally known as KAM theory (e.g. Tabor, 1989), describe how the nonsurviving tori break up to form chains of "islands" surrounded by a "chaotic sea" as seen in Fig. 2. For a large perturbation strength $\varepsilon$ all of the original tori will have been broken up, but the secondary islands that are formed in the process are robust and persist even when the magnitude of the perturbation exceeds that of the background flow. It has been shown (Brown, 1998; Beigie et al., 1991) that for multiply-periodic perturbations the situation is essentially the same as for perturbations with simple periodic time dependence. This follows from the observation that Eq. (1), with $\psi\left(x, y, \sigma_{1} t, \cdots, \sigma_{n} t\right)$ where $\sigma_{i} t$ is defined modulo $2 \pi$, can be transformed to an autonomous Hamiltonian system with a bounded phase space with $(n+1)$ degrees of freedom that is constrained by $n$ integrals. KAM theory (the KAM theorem and related results) applies to the transformed system, so phase space is generically partitioned into nonintersecting regular and chaotic regions. A Poincaré section could, in principle, be constructed for such a system by using a multiple slicing technique (cf. Parker and Chua, 1989 ) but slicing is practical only when $n=1$. The significance of the extension of KAM theory to multiply-periodic systems is that in the system defined by Eq. (1) with $\psi^{(1)}$ given by Eq. (2), phase space $(x, y)$ is expected to be partitioned into "regular islands" in an otherwise "chaotic sea." The numerical evidence presented in Figs. 3-6 supports this expectation.

The coexistence of regular and chaotic fluid particle trajectories in mesoscale and large-scale oceanic flows has been 


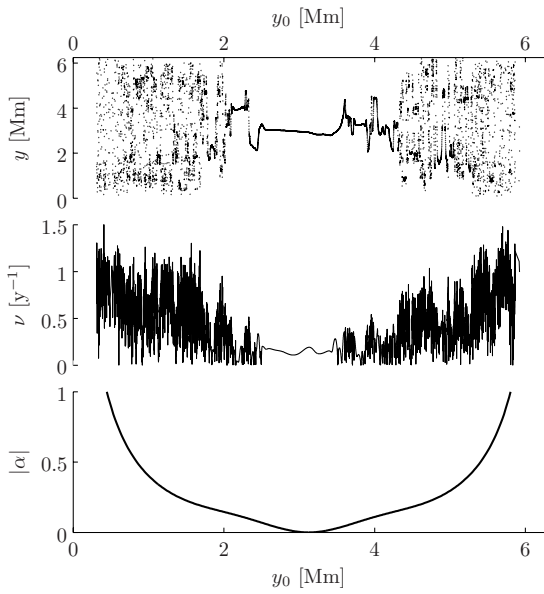

(a)

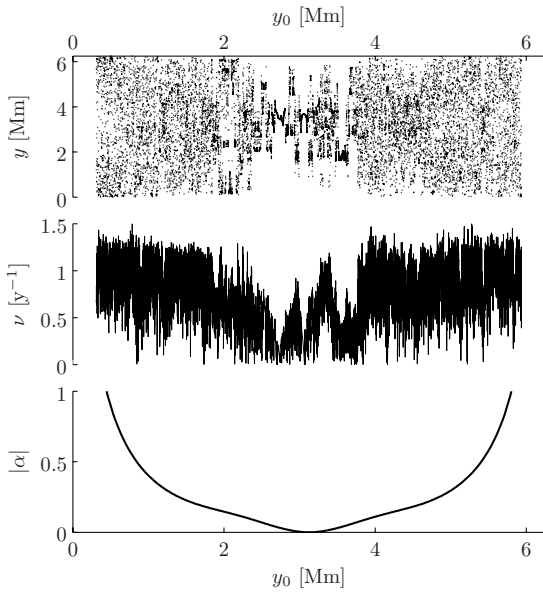

(b)

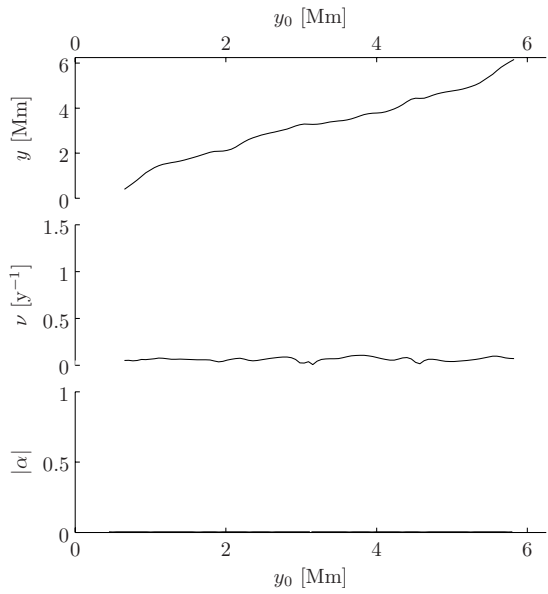

(c)

Fig. 3. Trajectory final meridional position $y$ (top panels), finite-time estimate of the Lyapunov exponent $v$ (middle panels), and absolute value of stability parameter $\alpha$ (bottom panel) as a function of initial meridional position. Background and perturbation fields are as in Fig. 1. All particles have initial longitudinal positions $x_{0}$ at the center of the background gyre. The integration time is $12 \mathrm{y}$ and $\Delta y_{0} \approx 5.5 \mathrm{~km}$.

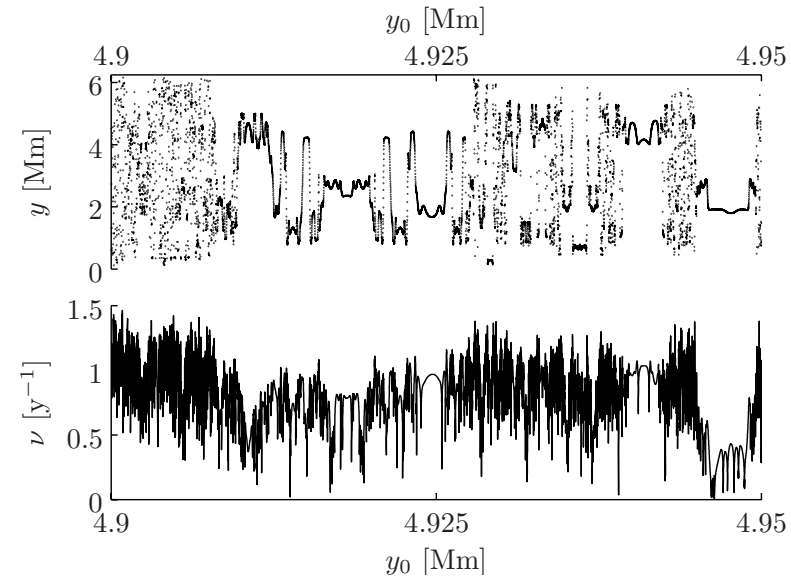

(a)
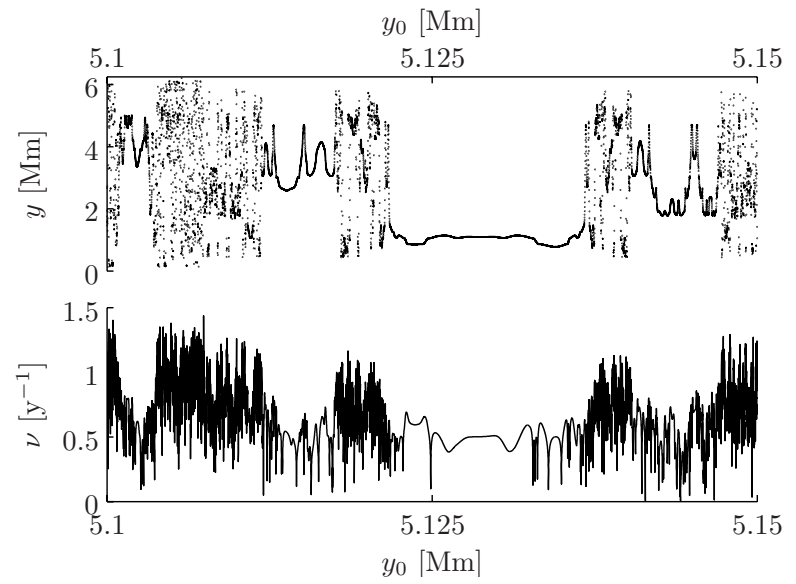

(b)

Fig. 4. Blow up of two portions of the top and middle panels of Fig. $3 \mathrm{~b}$ using $\Delta y_{0} \approx 5 \mathrm{~m}$.

suggested in some analyses of surface drifters and submerged floats (Osborne et al., 1986, 1989; Richardson et al., 1989; Brown and Smith, 1990). The preceding discussion provides an explanation of the underlying physics.

\section{Particle trajectory stability}

In this section we describe the important influence of the background flow on particle trajectory stability that was mentioned above in our discussion of Figs. 3a, c. The ideas presented here apply to any canonical Hamiltonian system in which the Hamiltonian consists of a superposition of an integrable component and a nonintegrable perturbation. Other applications are described in Beron-Vera and Brown (2003a, b).

The explanation of this behaviour makes use of the actionangle description of the motion of particles in the background flow (e.g. Abdullaev and Zaslavsky, 1991). Let

$I:=\frac{1}{2 \pi} \oint \mathrm{d} x Y\left(x ; \psi^{(0)}\right)$,

where $Y$ is the meridional coordinate of an isoline of $\psi^{(0)}$, be the action variable, and consider the canonical transformation $(x, y) \mapsto(\vartheta, I)$, defined implicitly by

$y=\partial_{x} G, \vartheta=\partial_{I} G, G(x, I):=\int \mathrm{d} x Y\left(x ; \psi^{(0)}\right)$, 


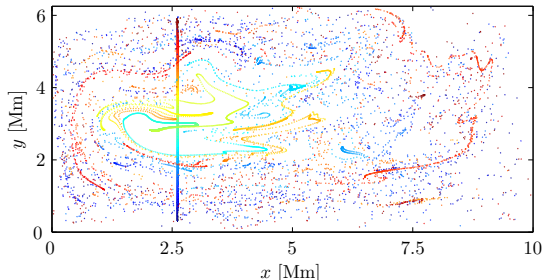

(a)

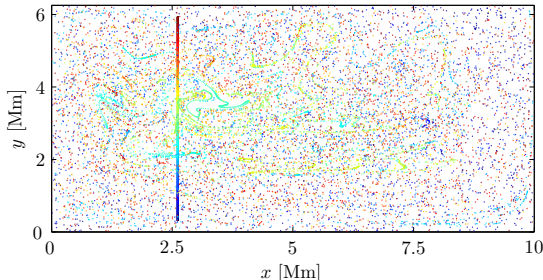

(b)

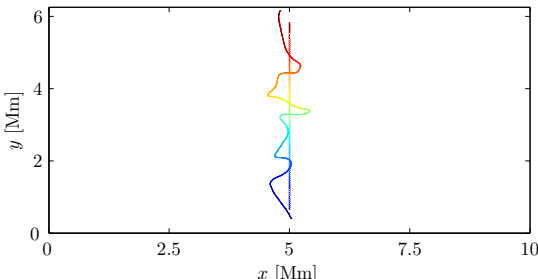

(c)

Fig. 5. Initial position (straight segment) and position after $12 \mathrm{y}$ of a discretely sampled $\left(\Delta y_{0} \approx 5.5 \mathrm{~km}\right)$ material line of fluid advected by the total (background plus perturbation) flows of Fig. 1. Colours indicate the initial meridional position of particles.

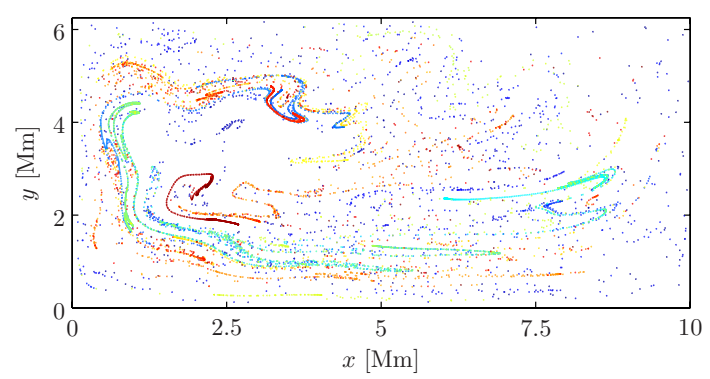

(a)

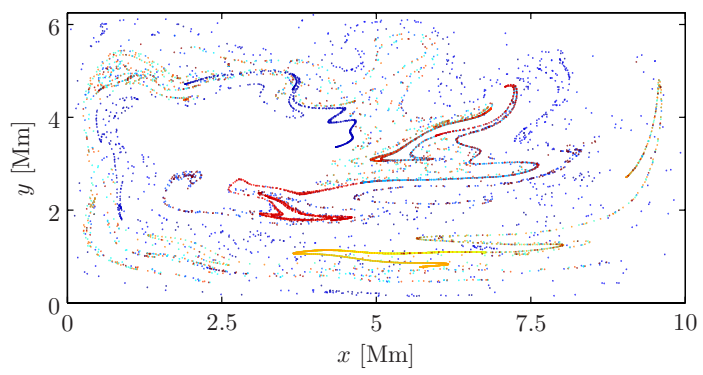

(b)

Fig. 6. Blow up of two portions of Fig. $5 \mathrm{~b}$ corresponding to trajectories shown in Figs. $4 \mathrm{a}$, b; here $\Delta y_{0} \approx 5 \mathrm{~m}$. Colours indicate the initial meridional position of particles; the initial material lines are so short that they appear as dots.

where $\vartheta$ is the angle variable. According to the above transformation,

$\psi(x, y, t) \mapsto \bar{\psi}^{(0)}(I)+\varepsilon \bar{\psi}^{(1)}(I, \vartheta, t)$

and Eqs. (1a) take the form

$\dot{I}=-\varepsilon \partial_{\vartheta} \bar{\psi}^{(1)}, \quad \dot{\vartheta}=\omega+\varepsilon \partial_{I} \bar{\psi}^{(1)}$,

where

$\omega(I):=\mathrm{d} \bar{\psi}^{(0)} / \mathrm{d} I$.

When $\varepsilon=0$, Eqs. (3), which have one degree of freedom, are autonomous and the corresponding Hamiltonian, $\bar{\psi}^{(0)}$, is an integral of motion that constrains the dynamics. As a consequence, the equations can be solved by quadratures and the motion is periodic with angular frequency $\omega$. Namely, $I=I_{0}$ and $\vartheta=\vartheta_{0}+\omega t \bmod 2 \pi$, where $I_{0}$ and $\vartheta_{0}$ are constants. Every solution curve is thus a line that winds around an invariant one-dimensional torus $\left\{I_{0}\right\} \times T^{1} \subset \mathbb{R} \times T^{1}$, whose representation in $(x, y)$-space is the closed curve given by the isoline $\psi^{(0)}=\bar{\psi}^{(0)}\left(I_{0}\right)$.

With the perturbation term, the corresponding Hamiltonian, $\bar{\psi}^{(0)}+\varepsilon \bar{\psi}^{(1)}$, is no longer an integral of motion (the equations are nonautonomous) and the system may be sensitive to initial conditions, thereby leading to chaotic motion. The distinction between regular and chaotic trajectories is commonly quantified by the Lyapunov exponent (e.g.
Parker and Chua, 1989), a measure of the rate at which neighboring trajectories diverge,

$v_{\infty}:=\lim _{t \rightarrow \infty} \frac{1}{t} \ln \left|v^{\mathbf{Q}}\right|$,

where $v^{\mathbf{Q}}(t)$ is the largest of the two eigenvalues of the socalled stability matrix $\mathbf{Q}(t)$, which is given by

$\mathbf{Q}:=\left[\begin{array}{cc}\partial_{I_{0}} I & \partial_{\vartheta_{0}} I \\ \partial_{I_{0}} \vartheta & \partial_{\vartheta_{0}} \vartheta\end{array}\right]$

Because of the area preservation property of Eqs. (1a) or (3) the product of the two eigenvalues of $\mathbf{Q}$ is unity, so there is no loss of generality in considering only the largest eigenvalue. Each column of $\mathbf{Q}$ corresponds to a vector perturbation $(\delta I, \delta \vartheta)$ to a trajectory in the nonautonomous system (3), and satisfies the so-called variational equations,

$$
\begin{aligned}
& \left(\begin{array}{c}
\delta \dot{I} \\
\delta \dot{\vartheta}
\end{array}\right)=\left[\begin{array}{cc}
0 & 0 \\
\omega^{\prime} & 0
\end{array}\right]\left(\begin{array}{l}
\delta I \\
\delta \vartheta
\end{array}\right) \\
& \quad+\varepsilon\left[\begin{array}{rr}
-\partial_{I \vartheta} \bar{\psi}^{(1)} & -\partial_{\vartheta \vartheta} \bar{\psi}^{(1)} \\
\partial_{I I} \bar{\psi}^{(1)} & \partial_{I \vartheta} \bar{\psi}^{(1)}
\end{array}\right]\left(\begin{array}{c}
\delta I \\
\delta \vartheta
\end{array}\right),
\end{aligned}
$$

where $\omega^{\prime}:=\mathrm{d} \omega / \mathrm{d} I$. Equations (7) and (3) constitute a system of four coupled equations.

Variational equations that describe the growth of perturbations using Cartesian coordinates $(\delta x, \delta y)$ have the same 
form as Eq. (7) except that in the Cartesian form all four elements of the first matrix on the r.h.s. of Eq. (7) are generally non-zero. Our numerical finite-time Lyapunov exponent estimates are based on the Cartesian equivalent of Eqs. (7) and (3), which is generally more convenient for numerical calculations. We have chosen to show the $(\delta I, \delta \vartheta)$ form of these equations to highlight the important role played by $\omega^{\prime}$. An example of a closely related study which does not exploit action-angle variables, and which consequently overlooks the critical importance of $\omega^{\prime}$, is Richards et al. (1995).

A simple but very important observation follows from the action-angle formalism. Dependence of both particle (3) and variational (7) equations on the background flow enters only through the function $\omega(I)$. Equations (7) strongly suggests that trajectory stability and $\omega^{\prime}$ are closely linked. The following heuristic argument explains the mechanism by which $\omega^{\prime}$ is expected to control trajectory stability. If one assumes that $\varepsilon$ is small and the second derivatives of $\bar{\psi}^{(1)}$ are zero-mean random variables, then when $\omega^{\prime}=0$ these terms should lead to slow (power-law) growth of $\delta \vartheta$ and $\delta I$. If $\left|\omega^{\prime}\right|$ is large, this term will cause a rapid growth of $|\delta \vartheta|$ for any non-zero $|\delta I|$. The perturbation terms will then lead to a mixing of $|\delta \vartheta|$ and $|\delta I|$. The term $\omega^{\prime}$ will lead, in turn, to further growth of $|\delta \vartheta|$. As this process repeats itself, both $|\delta I|$ and $|\delta \vartheta|$ are expected to grow rapidly. The role played by $\omega^{\prime}$ in this process is to amplify small perturbations caused by the second term on the r.h.s. of Eqs. (7). Thus, when $\varepsilon$ is small, trajectory instability is expected to be significantly enhanced when $\left|\omega^{\prime}\right|$ is large. When $\varepsilon$ is sufficiently large that the two terms on the r.h.s. of Eqs. (7) have comparable magnitude, the role played by $\left|\omega^{\prime}\right|$ in amplifying perturbations is expected to be less important. Increased trajectory instability should result in larger numerical estimates of Lyapunov exponents. A dynamicalsystems-based argument on the role of $\omega^{\prime}$ in controlling trajectory stability is given below; that argument is consistent with the above heuristic argument.

The lower panels of Fig. 3 show the absolute value of the stability parameter (Zaslavsky, 1998; Beron-Vera and Brown, 2003a, b)

$\alpha(I):=\frac{I}{\omega} \frac{\mathrm{d} \omega}{\mathrm{d} I}$

as a function of trajectory initial condition; recall that these initial conditions correspond to variable $y_{0}$ with $x_{0}$ fixed at the gyre center. Comparison of the middle and lower panels of Fig. 3a suggests that when the perturbation to the background steady flow is weak, trajectory instability increases, on average, with increasing $|\alpha|$. Figure $3 b$ shows that for a strong perturbation this trend is less strong, although the most stable trajectories are clearly those in the region of the flow where $|\alpha|$ is small. The background flow $\mathrm{R}$ used to produce Fig. 3c was chosen because it has the property $\alpha=0 \forall I$. Because the same perturbation flows were used to produce Figs. $3 \mathrm{a}$ and $3 \mathrm{c}$ the difference between these figures is entirely due to the difference in the background flows. The remarkable stability of trajectories in Fig. $3 \mathrm{c}$ is due to the property $\alpha=0 \forall I$ in flow $\mathrm{R}$. The same comment applies to the difference between Figs. 5a and 5c, which were produced using the same flows that were used to produce Figs. $3 \mathrm{a}$ and 3c. All of the aforementioned observations relating to Figs. 3 and 5 are consistent with the heuristic argument given in the preceding paragraph describing how $\left|\omega^{\prime}\right|$ is expected to control trajectory stability.

The physical interpretation of the stability parameter $\alpha$ is illustrated in Fig. 7. Figures 7a and b show the evolution of a material line of fluid in background flows $S$ and $R$, respectively. The material line is shown at $t=0$ and at $t=12 \mathrm{y}$. Also, shown is a plot of both $2 \pi / \omega$ and $|\alpha|$ as a function of $y_{0}$ (for $y_{0}>y_{\mathrm{C}}$, the meridional coordinate of the gyre center) in each environment. As a consequence of the uniqueness of solutions to Eq. (1) and continuity of the velocity field, the material line of fluid cannot break or intersect itself but it can increase in complexity with time. Because the motion in Fig. 7 is integrable (i.e. each point of the material line is constrained to lie on a surface of constant $I \forall t$ ) and because attention is restricted to background flows for which $\psi^{(0)}$ has compact and closed level sets, i.e. gyre flows, the length of the material line can grow with time, at most, following a power law. Background flow $\mathrm{R}$ has a special property. In that background flow the material line just rotates clockwise at a constant rate $\omega=\omega_{\mathrm{R}}\left(=2 \pi \mathrm{y}^{-1}\right)$, independent of $I$, so $\alpha=0 \forall I$. In contrast, $\omega$ varies with $I$ in background flow $\mathrm{S}$. The monotonic decay of $\omega$ as a function of $I$ in background flow $\mathrm{S}$ induces a shear in phase space which causes the outermost points of the material line to rotate more slowly than the innermost ones and, hence, causes the material line to spiral. In background flow $\mathrm{R}$ there is no shear. In polar coordinates radial shear can be defined as

$r \partial_{r}\left(r^{-1} u_{\theta}\right)$

where $r$ is the radial coordinate and $u_{\theta}$ is the $\theta$-component of the velocity field. More correctly, this quantity is twice the $r \theta$-component of the strain-rate tensor for rotational motion (e.g. Batchelor, 1964). The connection with motion in phase space can be accomplished by identifying $I$ with $r$ and $\omega I$ with $u_{\theta}$. The replacements $r \mapsto I$ and $u_{\theta} \mapsto \omega I$ in Eq. (9) thus give the analogous expression $I \omega^{\prime}$ for the shear in phase space. Notice that this expression is (apart from the $\omega^{-1}$-factor) the stability parameter $\alpha$. We have chosen to include the $\omega^{-1}$-factor in the definition of $\alpha$ because of precedent (Zaslavsky, 1998; Beron-Vera and Brown, 2003a, b) and because it is convenient to make $\alpha$ dimensionless.

To see the importance of the shear in the background flow, compare Figs. 7a and b with Figs. 5a and c, which show the evolution of the same initial material line segments in the total (background plus perturbation) flows. Notice the highly complicated structure of the segment in the perturbed flow $\mathrm{S}$ (Fig. 5a) as compared to that in the unperturbed one (Fig. 7a). (Note that the number of particles used to produce Fig. 5a is far too small to resolve what should be an unbroken smooth curve which does not intersect itself.) In contrast, observe that in environment $\mathrm{R}$ the perturbation has only a very minor effect on the evolution of the material line (Figs. 5c and 7b). 


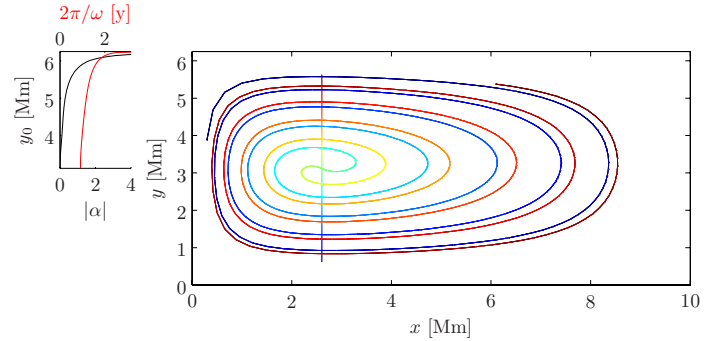

(a)

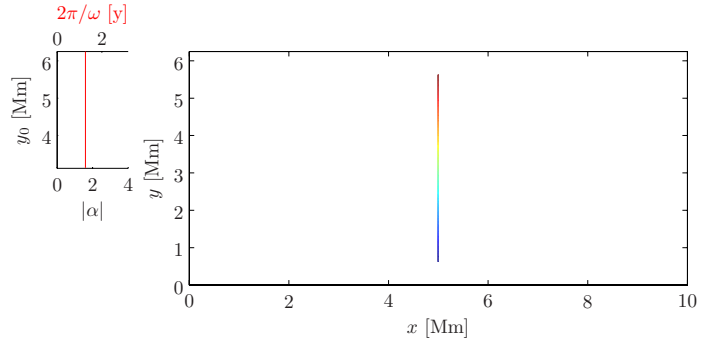

(b)

Fig. 7. Evolution of a material line of fluid in background flows $S$ (a) and R (b). Upper-left plots depict rotational period, $2 \pi / \omega$, and stability parameter, $\alpha$, as a function of initial meridional position.

Additional insight into why $\alpha$ should be expected to control trajectory stability comes from the following argument. The perturbation streamfunction $\varepsilon \psi^{(1)}$ has the effect of introducing perturbations to the action $I$ of a given particle by the amount $\delta I$. If $\delta I$ is assumed to be small and of the same order as the perturbation streamfunction $[\delta I=O(\varepsilon)$, say], then $\omega$ experiences the change

$\omega \mapsto(1+\alpha \delta I / I) \omega$

$+O\left(\varepsilon^{2}\right)$. The perturbation to $\omega$ depends on both the perturbation $\delta I$ and the background flow via $\alpha$. Under the change $I \mapsto I+\delta I$, a sufficient condition for $\omega$ to remain invariant at $O(\varepsilon)$ is $\alpha=0$. This provides an explanation for the remarkable stability of the particle trajectories in flow R. To $O(\varepsilon)$ a nonvanishing shear $(\alpha \neq 0)$ appears as a necessary condition to sustain the successive stretching and folding of the material line of fluid after it gets distorted by the perturbation. (Of course, chaotic motion is still possible when $\alpha=0$ provided that $\varepsilon$ is sufficiently large.) It is thus expected that where $|\alpha|$ is small (resp., large) there will be less (resp., more) sensitivity to initial conditions and, hence, the motion be more regular (resp., chaotic). Support for this conjecture is given in the numerical simulations presented in this paper.

Finally, the role of $\omega^{\prime}$ in dynamical systems theory deserves further comment. A nondegeneracy condition, $\omega^{\prime} \neq 0$, must be satisfied in order for the KAM theorem to apply and, hence, to guarantee that some trajectories are nonchaotic provided the strength of the time-dependent perturbation is sufficiently weak. This theorem does not imply, however, that trajectories are unstable when $\omega^{\prime}=0$; the KAM theorem does not address this limit. The mechanism that leads to chaos is the excitation of resonances at discrete frequencies. For a sufficiently strong perturbation, neighboring resonances overlap and chaotic motion results (e.g. Tabor, 1989). The width in frequency of each resonance is proportional to $\left|\omega^{\prime}\right|^{1 / 2}$, so one expects, on average, motion to become increasingly chaotic as $\left|\omega^{\prime}\right|$ increases. This expected trend is consistent with the arguments and numerical simulations that we have presented above. The trend toward increasingly chaotic motion with increasing $\left|\omega^{\prime}\right|$ does not, of course, rule out some nonchaotic motion for fixed but large $\left|\omega^{\prime}\right|$. Note also that the trends that we have described apply on average; details depend on details of the flow, both background and perturbation.

\section{Concluding remarks}

In this paper, we considered particle motion in unsteady incompressible two-dimensional flows consisting of a steady background gyre on which a highly structured unsteady wave-like perturbation is superimposed. The numerical simulations presented strongly suggest that: (i) phase space is mixed, characteristic of near-integrable one-and-a-halfdegree-of-freedom Hamiltonian systems; and (ii) particle trajectory stability strongly depends on the structure of the background (steady) component of the flow.

The mixed phase space structure, in which "islands" of stability emerge from an otherwise chaotic "sea," was explained as a consequence of the applicability of KAM theory. The mixed phase space provides an explanation for the occurrence of patches of poorly stirred fluid in a mostly vigorously stirred flow. Trajectory instability was shown to increase with increasing magnitude of $\alpha:=I \omega^{\prime} / \omega$, where $2 \pi / \omega(I)$ is the period of revolution of a particle in the background gyre flow and $I$ is the particle's action variable in the background flow.

These results provide important insight into the physics underlying Lagrangian ocean dynamics. In addition to this insight, the results described are potentially important in a variety of practical problems. The occurrence of Lagrangian "islands of stability" has important implications for the transport and dispersal of tracers ranging from nutrients to toxic pollulants. Knowledge that such "islands" are smaller and less abundant, on average, in regions of flows where $\left|\omega^{\prime}\right|$ is large might be exploited when deciding where to place a sewage outfall, for example.

Acknowledgement. The comments of an anonymous reviewer have led to improvements in the manuscript. This work has been supported by Code 321OA of the US Office of Naval Research. 
Edited by: S. Wiggins

Reviewed by: one referee

\section{References}

Abdullaev, S. S. and Zaslavsky, G. M.: Classical nonlinear dynamics and chaos of rays in wave propagation problems in inhomogeneous media, Usp. Phys. Nauk, 161, 1-43, 1991.

Arnold, V. I.: Mathematical Methods of Classical Mechanics, Springer, 2nd edn., 1989.

Batchelor, G. K.: An Introduction to Fluid Dynamics, Cambridge University, 1964.

Beigie, D., Leonard, A., and Wiggins, S.: Chaotic transport in the homoclinic and heteroclinic tangle regions of quasiperiodically forced two-dimensional dynamical systems, Nonlinearity, 4, 775-819, 1991.

Beron-Vera, F. J. and Brown, M. G.: Ray stability in weakly rangedependent sound channels, J. Acoust. Soc. Am., 114, 123-130, 2003a.

Beron-Vera, F. J. and Brown, M. G.: Travel time stability in weakly range-dependent sound channels, J. Acoust. Soc. Am., submitted (e-print nlin.CD/03 07 002) , 2003b.

Brown, M. and Smith, K.: Are SOFAR float trajectories chaotic?, J. Phys. Oceanogr., 20, 139-149, 1990.

Brown, M. G.: Phase space structure and fractal trajectories in $1 \frac{1}{2}$ degree of freedom Hamiltonian systems whose time dependence is quasiperiodic, Nonlin. Proc. Geophys., 5, 69-74, 1998.
Malhotra, N., Mezić, I., and Wiggins, S.: Patchiness: A new diagnostic for Lagrangian trajectory analysis in time-dependent fluid flows, Int. J. Bif. Chaos, 8, 1053-1093, 1998.

Osborne, A. R., Kirwan, A. D., Provenzale, A., and Bergamasco, L.: A search for chaotic behaviour in large and mesoscale motions in the pacific ocean, Physica, 23D, 75-83, 1986.

Osborne, A. R., Kirwan, A. D., Provenzale, A., and Bergamasco, L.: Fractal drifter trajectories in the kuroshio extension, Tellus, 41 A, 416-435, 1989.

Parker, T. S. and Chua, L. O.: Practical Numerical Algorithms for Chaotic Systems, Springer, 1989.

Pasmanter, R.: Anomalous diffusion and anomalous stretching in vortical flows, Fluid Dyn. Res., 3, 320-326, 1988.

Richards, K. J., Jia, Y., and Rogers, C. F.: Dispersion of tracers by ocean gyres, J. Phys. Oceanogr., 25, 873-887, 1995.

Richardson, P. L., Walsh, D., Armi, L., Schröder, M., and Price, J. F.: Tracking three meddies with SOFAR floats, J. Phys. Oceanogr., 19, 371-383, 1989.

Stommel, H.: The Gulf Stream, University of California, 2nd Ed., 1966.

Tabor, M.: Chaos and Integrability in Nonlinear Dynamics, John Wiley and Sons, 1989.

Zaslavsky, G. M.: Physics of Chaos in Hamiltonian Systems, Imperial College, 1998. 